\title{
Effective Transport Properties Accounting for Electrochemical Reactions of Proton-Exchange Membrane Fuel Cell Catalyst Layers
}

\author{
Jon G. Pharoah ${ }^{\mathrm{a},{ }^{*}, \text { Hae-Won Choi }}{ }^{\mathrm{a}}$, Chih-Che Chueh ${ }^{\mathrm{a}}$, David B. Harvey ${ }^{\mathrm{b}}$ \\ ${ }^{a}$ Queen's-RMC Fuel Cell Research Centre, \\ 945 Princess St., Kingston, ON, K7L 5L9, Canada \\ ${ }^{b}$ Ballard Power Systems, \\ 9000 Glenlyon Parkway, Burnaby, BC, V5J 5J8, Canada \\ *Email: pharaoh@me.queensu.ca
}

\begin{abstract}
There has been a rapidly growing interest in three-dimensional microstructural reconstruction of fuel cell electrodes so as to derive more accurate descriptors of the pertinent geometric and effective transport properties. Due to the limited accessibility of experiments based reconstruction techniques, such as dual-beam focused ion beamscanning electro microscopy or micro X-Ray computed tomography, within sample micro-structures of the catalyst layers in polymer electrolyte membrane fuel cells (PEMFCs), a particle based numerical model is used in this study to reconstruct sample microstructure of the catalyst layers in PEMFCs. Then the reconstructed sample structure is converted into the computational grid using body-fitted/cut-cell based unstructured meshing technique. Finally, finite volume methods (FVM) are applied to calculate effective properties on computational sample domains.
\end{abstract}

\section{Introduction}

The catalyst layer is the seat of the electrochemical reactions taking place in a protonexchange membrane fuel cell (PEMFC). It is a complex porous medium made of networks of Carbon chains supporting platinum $(\mathrm{Pt})$ particles and covered with a layer of Ionomer (e.g. Nafion). In order to model a complete PEMFC, it is common to consider the catalyst layer as a homogeneous medium with effective properties that take into account the effect of the catalyst morphology on transport properties.

A micro-structural model of the catalyst layer of PEMFCs has been developed in order to investigate the interplay between composition, morphology and effective transport properties. Due to the limited accessibility of experiments based reconstruction techniques, such as dual-beam focused ion beam-scanning electro microscopy (FIB-SEM) or micro XRay computed tomography (XCT) $(6,7,8)$, within sample micro-structures of the catalyst layers in polymer electrolyte membrane fuel cells (PEMFCs), sample microstructures of catalyst layers are often reconstructed through numerical model $(1,2,3)$. Overlapping spheres of different diameters represents particles of Carbon, $\mathrm{Pt}$ and Ionomer. First forming random Carbon chains, then depositing Pt particles on these chains, and eventually covering the Carbon-Pt networks with Ionomer particles create the sample geometry.

A set of numerical tools has been developed to characterize and validate the morphology of the reconstructed catalyst layer sample, including the measurements of the electrochemical reaction site, percolating networks and the pore size distribution. Two independent methods were developed in order to calculate the effective transport properties: 
a finite-volume method (FVM) and a random-walk (RW) simulation. The FVM is implemented through the open source computational fluid dynamics (CFD) toolkit, OpenFOAM $^{\circledR}$ (4). The FVM follows a continuum approach that does not capture all the phenomena involved in diffusion in the gas phase. To complement a continuum based FVM, a RW simulation is applied in the transition diffusion regime. Indeed, the size of the catalyst pores is sufficiently small so that gas diffusion occurs in the transition regime, i.e. gas transport is affected by the frequent collisions between gas molecules and solid walls.

We will present results for effective transport properties for the electron- conducting phase (Carbon-Pt region), the ion-conducting phase (Ionomer region) and the gas diffusing pore phase (Pore space). The effect of the composition of the catalyst layers on effective transport properties accounting for electrochemical reactions will be addressed. We will show the importance of taking into account the effect of pore confinement (Knudsen number) on gas diffusion and how these effects can be integrated into the FV solver.

\section{Model Descriptions}

\section{$\underline{\text { Sample Structure Generation }}$}
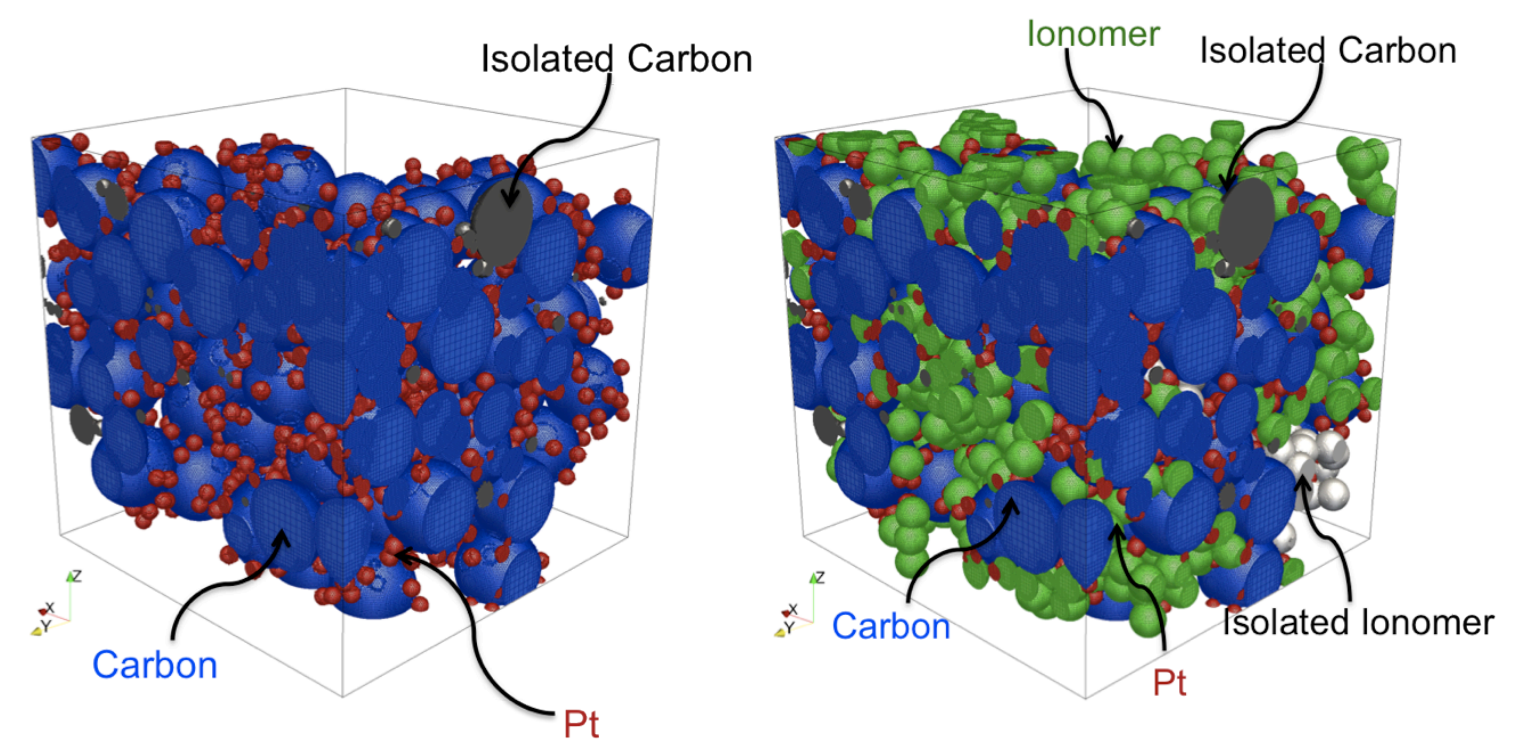

Figure 1: Sample reconstructed catalyst layer microstructure consisting of pseudo- randomly placed particles.

A particle based numerical method is used to generate pseudo-random sample microstructures for catalyst layers of PEMFCs. Example sample reconstruction is shown in Figure 1. As shown in Figure 1 (left), the first Carbon particle is placed in domain, children Carbon particles are repeatedly placed adjacent to their parent to form chained Carbon network, and Pt particles are deposited on Carbon network. Then Ionomer particles are deposited as spheres covering the Carbon/Pt network structure as given in Figure 1 (right). The Ionomer particles, which establish their own percolating network, conform to Carbon/Pt particles in order to serve the reaction site of catalyst layers in PEMFCs. Thus the sample domain consists of three phase regions: electron-conducting (Carbon/Pt) region, ionconducting (Ionomer) region, and gas transporting pore region. In this study, Carbon particle diameter of $25 \mathrm{~nm}$, Pt particle diameter of $5 \mathrm{~nm}$, and Ionomer particle diameter of $10 \mathrm{~nm}$ are selected for reconstructions. All particles are assumed as monosized. The sample domain 
size of $125 \mathrm{~nm} \times 125 \mathrm{~nm} \times 125 \mathrm{~nm}$, which is discretized with a body-fitted/cut-cell based unstructured grid, is considered. Computational sample domains, taking the coordinate and diameter information as input parmaeters, are constructed through MicroFOAM (3).

\section{Governing Equations}

The model incorporates underlying transport equations for electrons, ions (protons), oxygen and water vapor. Steady-state conditions are assumed and species transport is assumed as diffusion process for each phase. The electrochemical reactions are considered through the Butler-Volmer equation for a given fixed temperature at the interfaces between $\mathrm{Pt}$ and Ionomer surfaces. The effects of liquid water are not considered in this study.

$$
\begin{gathered}
\nabla \cdot\left(\sigma_{\text {elec }} \nabla \phi_{\text {elec }}\right)=-j \\
\nabla \cdot\left(\sigma_{\text {ion }} \nabla \phi_{\text {ion }}\right)=j \\
\nabla \cdot\left(D_{O_{2}} \nabla C_{O_{2}}\right)=-\frac{j}{4 F} \\
\nabla \cdot\left(D_{H_{2} O} \nabla C_{H_{2} O}\right)=\frac{j}{2 F} \\
j=i_{0} \frac{C_{O_{2}}}{C_{O_{2, \text { ref }}}}\left\{\exp \left(\alpha_{a} \frac{F \eta}{R T}\right)-\exp \left(-\alpha_{c} \frac{F \eta}{R T}\right)\right\}
\end{gathered}
$$

where $\sigma_{\text {elec }}$ is the electronic conductivity for the electron $(\mathrm{Carbon} / \mathrm{Pt})$ phase, $\sigma_{\text {ion }}$ is the ionic conductivity for the ion (Ionomer) phase, $D_{\mathrm{O}_{2}}$ is the oxygen diffusivity, $D_{\mathrm{H}_{2} \mathrm{O}}$ is the water vapor diffusivity, $\phi_{\text {elec }}$ is the electronic potential filed, $\phi_{\text {ion }}$ is the ionic potential field, $C_{\mathrm{O}_{2}}$ is the oxygen concentration field, $i_{0}$ is the reference exchange current density, $C_{O_{2} \text {, ref }}$ is the reference oxygen concentration field, $F$ is the Faraday's constant, $R$ is the universal gas constant, $T$ is the reference temperature of the electrode, $\alpha_{a}$ denotes anode charge transfer coefficient, $\alpha_{c}$ denotes cathode charge transfer coefficient and $\eta=\phi_{\text {elec }}-\phi_{\text {ion }}$ is the overpotential difference. The detail information for above mentioned parameters could be found in (1).

\section{Numerical Methods and Electrochemical Reactions}

The computations of Equations [1-5] are implemented by finite volume methods through the open source CFD toolbox, OpenFOAM ${ }^{\circledR}$ (4). Note that Equations [1-4] are coupled through electrochemical kinetic expression of Equation [5]. This coupled physics is implemented through the electrochemical boundary conditions given at the interfaces between $\mathrm{Pt}$ and Ionomer surfaces. The calculations of Equations [1-4] are iteratively evaluated by each independent Laplace equation with specified boundary conditions until they satisfy the convergence of solutions. In each iteration, the electrochemical reaction rate is updated based on Equation [5] and then this reaction rate is repeatedly plugged into Equations [1-4] as source (or sink) flux boundary conditions on the reaction site (i.e. the interface between Pt and Ionomer surfaces). 


\section{Effective Transport Properties and Knudsen Gas Diffusivity}

Effective transport properties, i.e., effective electronic/ionic conductivity and effective binary gas diffusivity, can be easily extracted from the solutions of Equations [1-4], followed by the procedure proposed in (3). On the other hand, the effective Knudsen gas diffusivity can be calculated provided that the characteristic length scale of pore confinement is known for the microstructure (5). The characteristic length scale of microstructure can be evaluated by the pore space geometry evaluation or by the Random walk (RW) simulation run on the microstructure. Once the characteristic length of pore confinement (i.e. $d_{c l}$ ), evaluated by either geometrically or statistically, is known, one can calculate the Knudsen number using the relationship such that $K n=\langle\lambda\rangle / d_{c l}$ where $\langle\lambda\rangle$ denotes the mean free path. Then the effective binary diffusivity including the Knudsen diffusion effects is evaluated by Bosanquet's formula such that

$$
\frac{D^{e f f}(K n)}{D_{b}}=\frac{\epsilon}{\tau} \frac{1}{1+K n}
$$

where $\epsilon$ is the porosity and $\tau$ denotes the tortuosity factor. $D^{\text {eff }}(K n)$ Is the effective gas diffusivity including the Knudsen diffusion effects where as $D_{b}$ is the bulk binary diffusivity.

\section{Numerical Results}

In order to calculate the effective transport properties, one needs to solve the coupled Equations [1-4] where Butler-Volmer Equation [5] is used as the constraint of Equations [14]. Figure 2 demonstrates FVM simulation examples for the electron potential, ion potential, and gas phase concentration fields. The parametric study performed in this study requires the hundreds of sample constructions along with the simulated solutions of their microstructures. The sample microstructures per each parameter composition are reconstructed using the particle based catalyst layer sample generation algorithm written in $\mathrm{C}$ language. Then each reconstructed sample catalyst layer is converted into a finite volume (FV) based bodyfitted/cut-cell grid using MicroFOAM (3). All simulations are performed on supercomputers available in the facilities of the Shared Hierarchical Academic Research Computing Network (SHARCNET: www.sharcnet.ca).
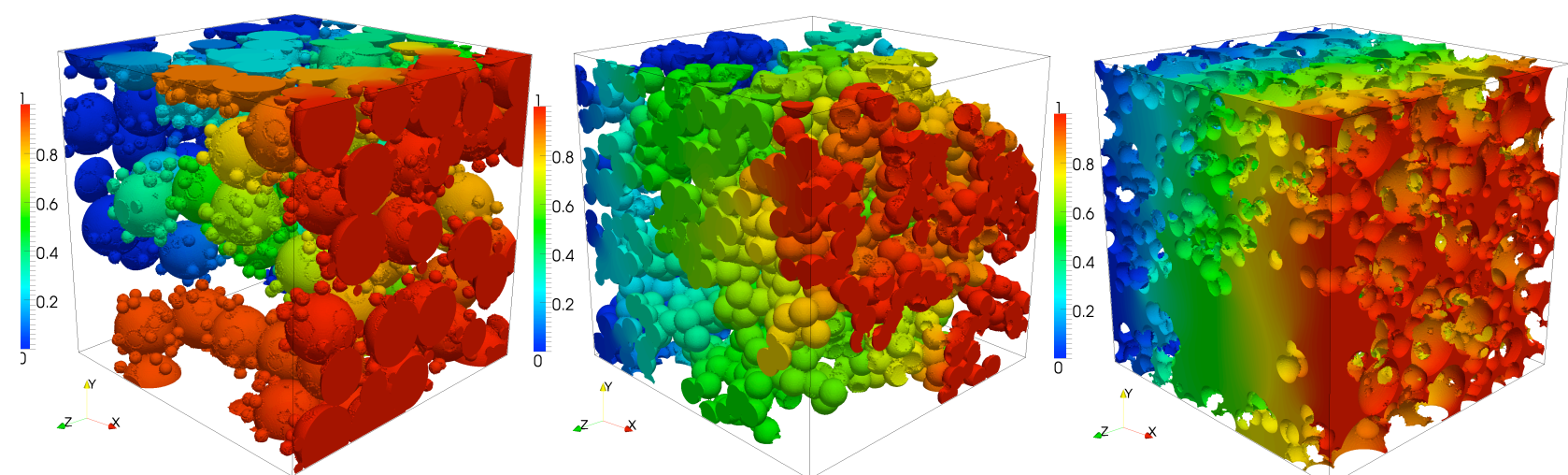

Figure 2: FVM simulation examples for the electron potential (left), ion potential (middle), and oxygen concentration (right) fields performed on a reconstructed catalyst layer sample. A reconstructed catalyst layer sample domain of $125^{3} \mathrm{~nm}^{3}$ is used here-in. 
Figure 3 shows the normalized effective electronic and ionic conductivities as a function of each phase volume fraction in a given sample domain. The effective electronic conductivity is in relatively good agreement to Koh-Fortini's empirical relationship, i.e. $k^{\text {eff }} / k=\epsilon_{\text {elec }} / 1+11\left(1-\epsilon_{\text {elec }}\right)^{2}$, as given in (9). Here $\epsilon_{\text {elec }}$ is the electron volume fraction. However, due to the relatively large Carbon particle diameter (i.e. $d_{\text {Carbon }}=25 \mathrm{~nm}$ ) per a given sample domain size (i.e. $125^{3} \mathrm{~nm}^{3}$ ), the normalized effective electronic conductivity suffered large variance. On the other hand, the normalized effective ionic conductivity has small variance thanks to relatively small Ionomer diameter (i.e. $d_{\text {Ionomer }}=10 \mathrm{~nm}$ ) per a given sample domain size. The normalized effective ionic conductivity follows the curve-fit correlation of $k^{\text {eff }} / k=1.24\left(\epsilon_{\text {ion }}-0.03\right)^{2.2}$. Note that there is no empirical or theoretical correlation is available for the effective Ionomer conductivity for catalyst layers of PEMFCs. Hence the direct validation for the effective ionic conductivity is not possible.
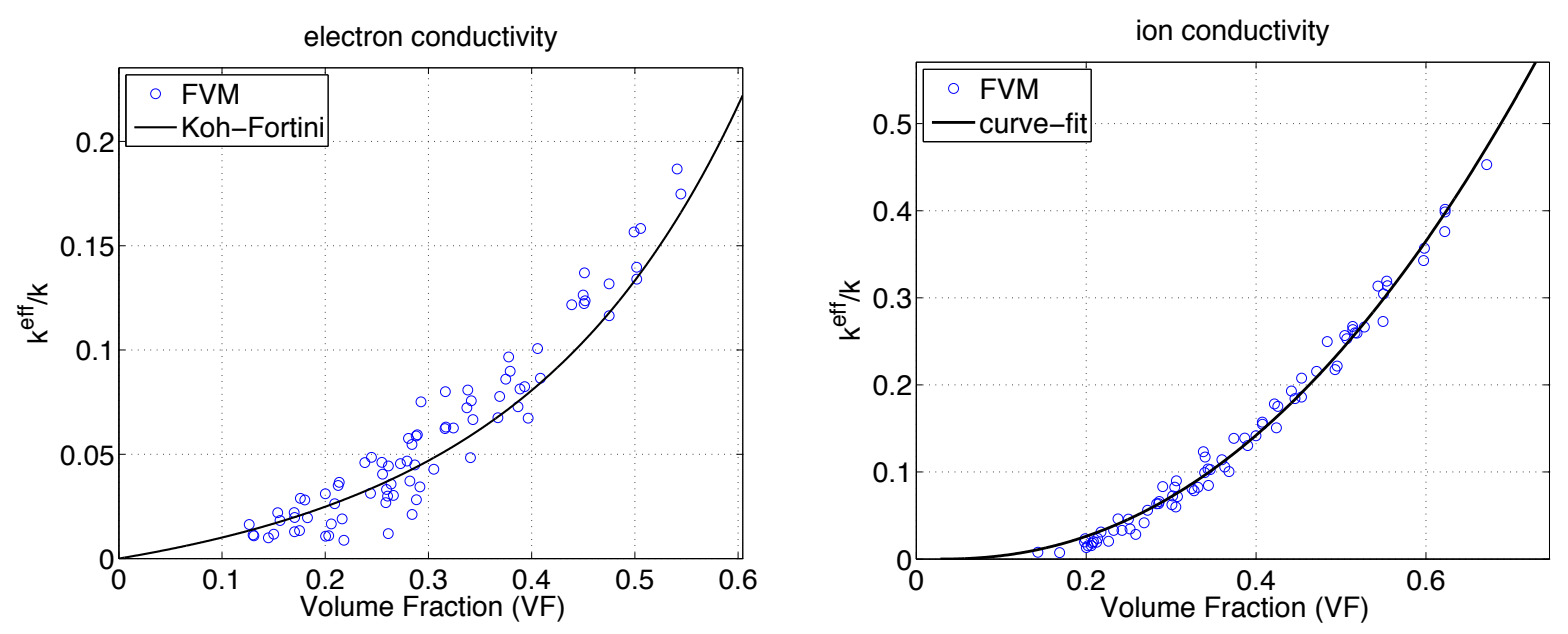

Figure 3: Normalized effective electronic (left) and ionic (right) conductivities as a function of each phase volume fraction in a sample domain.

Figure 4 summarizes the normalized effective gas diffusivity as a function of porosity of a given sample domain. No Knudsen effects are considered here-in. The effective gas diffusivity in this study is compared against the Bruggeman equation (i.e. $D^{\text {eff }} / D_{b}=\epsilon^{1.5}$ ) as well as the correlation from RW simulation proposed in (5). The normalized effective gas diffusivity in present work is in good agreement to the correction by RW simulation (5). However, our model results exhibited large variance due to its relatively small sample domain size (i.e. $0.125^{3} \mu \mathrm{m}^{3}$ ) used in this study. The normalized effective gas diffusivity in this study follows the curve-fit correlation of $D^{\text {eff }} / D_{b}=1.07(\epsilon-0.012)^{1.6}$.

Figure 5 demonstrates the Knudsen number (left) and normalized effective Knudsen gas diffusivity (right) as a function of porosity of a given sample domain. Knudsen number $\left(K n=\langle\lambda\rangle / d_{c l}\right)$ is the ratio between the mean free path (i.e., $\left.\langle\lambda\rangle\right)$ and characteristic length of pore confinement (i.e., $d_{c l}$ ), where the latter is calculated from the geometrical evaluation in this study. Alternatively, the characteristic length of pore confinement can also be evaluated by RW simulation, proposed in (10). The higher porosities result in lower Knudsen numbers. Due to very small size of particle diameters (such as Pt or Ionomer) within Catalyst layers, Knudsen numbers range in between transition regime and Knudsen regime. Therefore, it has found that the effective Knudsen gas diffusivity is much smaller than the effective gas diffusivity without Knudsen diffusion effects as shown in Figure 5 (right). 


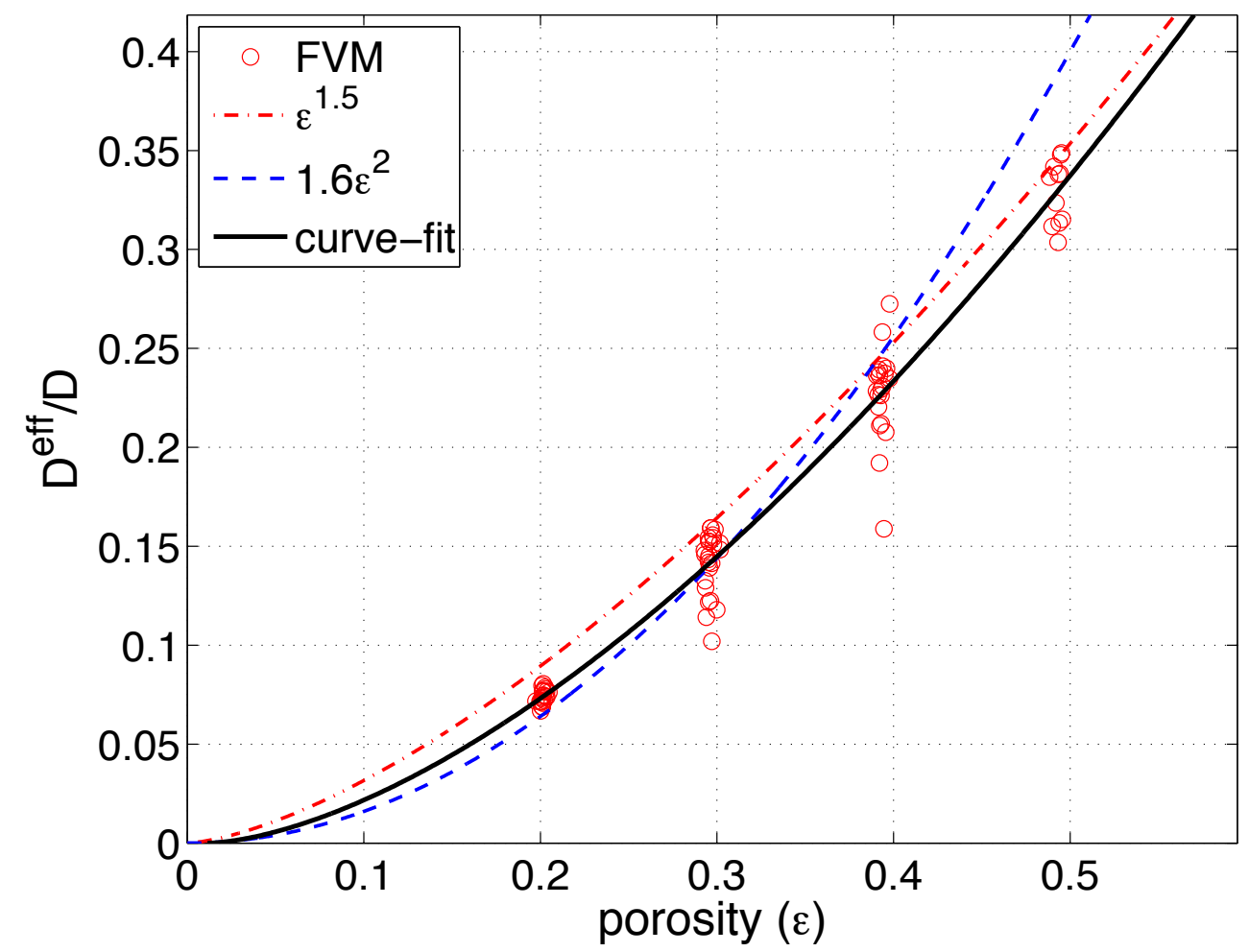

Figure 4: Normalized effective gas diffusivity as a function of porosity (pore phase volume fraction of a sample domain). No Knudsen diffusivity is considered herein.
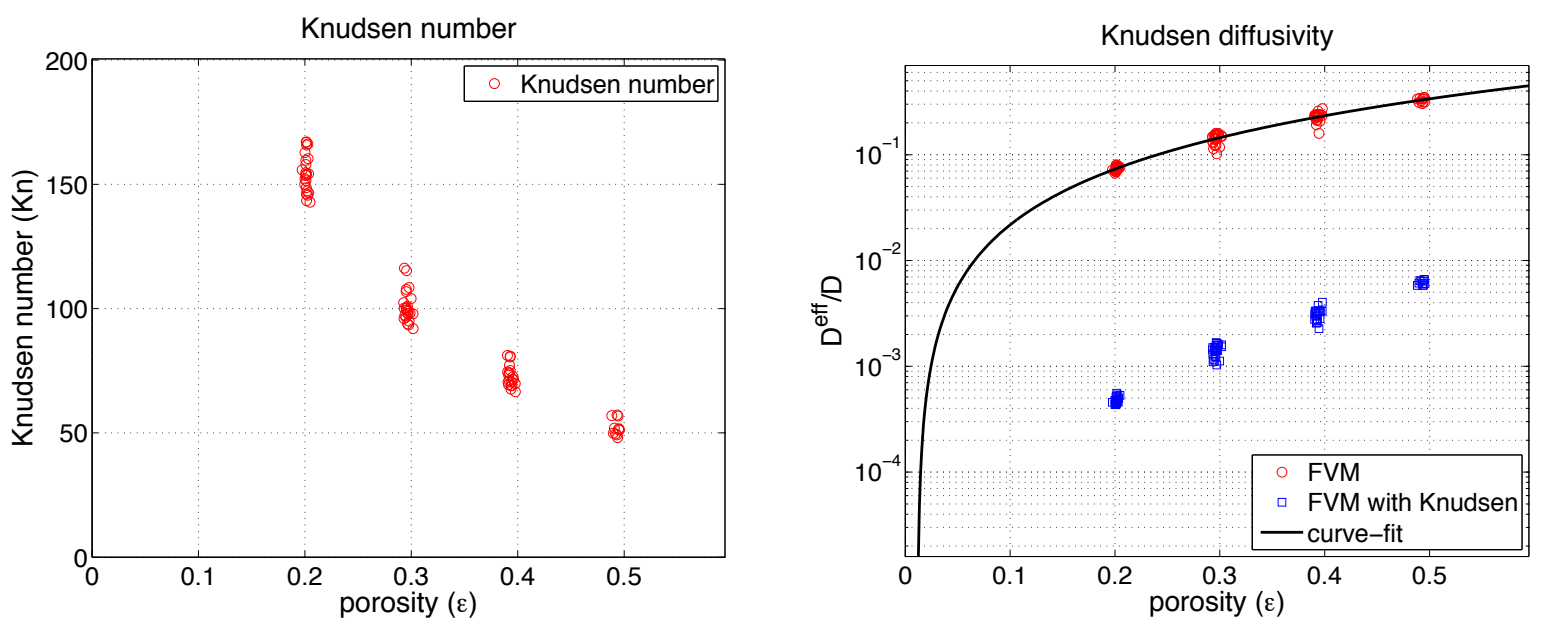

Figure 5: Knudsen number (left) and normalized effective Knudsen gas diffusivity (right) as a function of porosity (pore phase volume fraction of a sample domain).

\section{Conclusion}

In this study, we constructed the microstructure of catalyst layers used in PEMFCs. The reconstruction of microstructure is based on particle based a pseudo-random numerical model consists of overlapping Carbon, Pt, and Ionomer particles. This reconstructed 
microstructure was converted into a 3-D body-fitted/cut-cell based unstructured FV grid using MicroFOAM (3). Performing on the constructed sample computational domain, the effective transport properties were evaluated by using FVM through the open-source CFD toolbox, OpenFOAM ${ }^{\circledR}$. The effective electronic/ionic conductivity and gas diffusivity were evaluated as a function of each phase volume fraction. Finally, the effective Knudsen gas diffusivity was calculated by Bosanquet's formula and they were functions of Knudsen number $(K n)$. The higher porosities result in lower Knudsen numbers. It has found that the Knudsen numbers range in between transition regime and Knudsen regime. Therefore, it has found that the effective Knudsen gas diffusivity was much smaller than the effective gas diffusivity without Knudsen diffusion effects.

\section{References}

1. K.J. Lange, P.-C. Sui, N. Djilali, J. Power Sources, 196, 3195-3203 (2011).

2. T. Hattori, A. Suzuki, R. Sahnoun, M. Koyama, H. Tsuboi, N. Hatakeyama, A. Endou, H. Takaba, M. Kubo, C.A. Del Carpio, A. Miyamoto, Appl. Surf. Sci., 254, 7929-7932 (2008).

3. H.-W. Choi, A. Berson, J.G. Pharoah, S.B. Beale, Proc. IMechE PartA: J. Power Energy, 225, 183-197 (2011).

4. OpenFOAM ${ }^{\circledR}$ (Open Field Operation and Manipulation). The open source CFD toolbox available from http://www.openfoam.com.

5. A. Berson, H.-W. Choi, J.G. Pharoah, Physics Rev. E., 83, 026310 (2011).

6. H. Ostadi, P. Rama, Y. Liu, R. Chen, X.X. Zhang, K. Jiang, J. Membrane Sci., 351, 69-74 (2010).

7. A. Pfrang, D. Veyret, F. Sieker, G. Tsotridis, Int. J. Hydro. Energy, 35, 3751-3757 (2010).

8. S. Thiele, R. Zongerie, C. Ziegler, Nano Res., Published online (6 May 2011).

9. J.C.Y. Koh, A. Fortini, Int. J. Heat Mass Transfer, 16, 2013-2022 (1973).

10. D. Blore, Master's Thesis, Department of Mechanical and Materials Engineering, Queen's University, Kingston, ON, Canada (2011). 